\title{
Expression and Purification of Recombinant Mayaro Virus Envelope Glycoproteins E1 and E2 to Develop a Mayaro Virus Detection System
}

\author{
So Yeon $\mathrm{Yi}^{1,2}$, Kyungah Yoon ${ }^{3}$, Jungsun Kwon ${ }^{1}$, Kyoon Eon Kim², Kyoungsook Park ${ }^{1,4 *}$, Yong Beom Shin ${ }^{1,5 *}$ \\ ${ }^{1}$ BioNano Health Guard Research Center, 125 Gwahak-ro, Yuseong-gu, Daejeon 34141, Republic of Korea \\ 2Department of Biochemistry, College of Natural Sciences, Chungnam National University, 99 Daehak-ro, \\ Yuseong-gu, Daejeon 34134, Republic of Korea \\ ${ }^{3}$ Department of Clinical Laboratory Science, Daejeon Health Institute of Technology, Daejeon 34504, Republic of Korea \\ ${ }^{4}$ Department of General Education, Daejeon Health Institute of Technology, Daejeon 34504, Republic of Korea \\ ${ }^{5}$ Bionanotechnology Research Center, Korea Research Institute of Bioscience and Biotechnology (KRIBB), 125 \\ Gwahak-ro, Yuseong-gu, Daejeon 34141, Republic of Korea
}

\author{
Corresponding \\ Kyoungsook Park \\ Yong Beom Shin \\ Phone : $\begin{array}{r}+82-42-860-4445 \\ +82-42-860-4449\end{array}$
E-mail : marsp@kribb.re.kr.
ybshin@kribb.re.kr.
}

Received : January 09, 2020

Revised : March 02, 2020

Accepted : March 04, 2020

No potential conflict of interest relevant to this article was reported.

Copyright (C) 2020 Journal of Bacteriology and Virology

(C) This is an Open Access article distributed under the terms of the Creative Commons Attribution Non-Commercial License (http://creativecommons.org/ license/by-nc/3.0/)
Mayaro virus (MAYV) is a mosquito-transmitted alphavirus that produces an acute, usually non-fatal, febrile illness including Mayaro fever. Like other alphaviruses, the MAYV E1 and E2 envelope glycoproteins are major viral surface antigens that play a key role in host recognition and infection. Here, we report expression and purification methods for recombinant MAYV E1 (rE1) and rE2 using a baculovirus system. Enzyme-linked immunosorbent assays (ELISA) revealed that $\mathrm{rE} 1$ and $\mathrm{rE2}$ were antigenic and reacted with human anti-MAYV IgG and IgM. Cross-reactivity was also confirmed with human anti-Chikungunya virus (CHIKV) IgG and IgM. Furthermore, we developed an immunochromatographic strip test (IST) with rE2 to diagnose MAYV infection. Thus, purified rE2 may be valuable tool for rapidly diagnosing MAYV infection.

Key Words: Baculovirus/insect cell system, recombinant envelope glycoprotein 1 (rE1), recombinant envelope glycoprotein 2 (rE2), immunochromatographic strip test (IST), Mayaro virus (MAYV)

\section{INTRODUCTION}

Mayaro virus (MAYV) was found on the island of Trinidad and has caused small outbreaks in several parts of tropical South America. MAYV is a single strand positive RNA virus of the alphavirus genus in the family Togaviridae and is similar to chikungunya virus (CHIKV) (1-3). Like other alphaviruses, MAYV is an arbovirus (arthropod-borne virus) which is mostly transmitted by Haemagogus mosquitoes. Recent studies have shown that Aedes aegypti mosquitos can spread MAYV worldwide (4-6).

MAYV infection causes a CHIKV-like, acute, usually non-fatal, febrile illness with a 3-5 day duration that includes fever (known as Mayaro fever), joint pain, headache, rashes, and muscle ache. Some patients develop persistent, terrible joint pain that lasts up to a year after infection $(7,8)$. MAYV infection can be confirmed by viral isolation, RT-PCR, and serological diagnosis using hemagglutination 
inhibition tests and enzyme-linked immunosorbent assays (ELISA); however, these methods are unresponsive for a great number of infectious samples and live virus analysis may require biosafety laboratories. Furthermore, due to the antigenic similarity of MAYV and CHIKV, there is an acute need to develop more specific and precise methods for diagnosing MAYV infection (9).

The MAYV genome is a $12 \mathrm{kbp}$ long single-stranded RNA containing two open reading frames (ORFs). It encodes four non-structural proteins (nsP1, nsP2, nsP3, and nsP4) and five structural proteins (C, E3, E2, 6K, and E1). The structural envelope glycoproteins E1 and E2 are imbedded in the envelope on the viral surface. E1 mediates the fusion of the virus with the host cell, while E2 is mainly involved in attaching viruses to host cells (10). As for alphaviruses, E1 and E2 are targets of the anti-MAYV antibody response; therefore, E1 and E2 would be useful targets for immunodiagnostic analysis

In this study, we explain the generation of soluble MAYV E1 and E2 using a baculovirus/insect expression system. Recombinant envelope proteins E1 ( $r E 1)$ and $r E 2$ were successfully expressed and purified in soluble forms, with rE2 better expressed and more stable than $\mathrm{rE}$. ELISA revealed that $\mathrm{rE} 1$ and $\mathrm{rE2}$ were antigenic and reactive for specifically detecting human anti-MAYV IgG or IgM. Cross-reactivity was also evaluated using human anti-CHIKV $\lg G$ or $\lg \mathrm{M}$. In particular, rE2 displayed a higher ELISA value than rE1. Furthermore, we developed an immunochromatographic strip test (IST) using rE2 to detect human anti-MAYV IgG or IgM, demonstrating the potential applicability of rE2 in a diagnosis system to detect MAYV infection.

\section{MATERIALS AND METHODS}

\section{Construction of recombinant expression vector containing MAYV envelope glycoproteins E1 and E2}

Synthetic MAYV DNA encoding E1 (GenBank \# KT818520, residues 1 442) or E2 (GenBank \# KT818520, residues 1 421) and a C-terminal six histidine tag (His-tag) were obtained from Bioneer. Two enzyme sites, Smal and Xbal, were created at the 5' and 3 ' ends of the $E 1$ and $E 2$ genes, respectively. $E 1$ and $E 2$ were amplified by PCR using the following primers: E1F, 5'-CCCGGGGGATCCTACGAGCACACG-3'; E1R, 5'-TCTAGATTGCG CCCAAGTCATTGCAGTGCTGGATA-3'; and E2F, 5'CCCGGGGGATCCAGCACTGC AAAT-3'; E2R， 5'-TCTAGACGTAGGATGCAGTCCGTAGTAGTATTC-3' (restriction sites underlined). The PCR products were cloned into corresponding sites in pAcGP67A (Pharmingen). The E1 and E2 genes inserted downstream of the robust polh promoter to create pAcGP67A-E1 and pAcGP67A-E2.

\section{Insect cell culture and transfection}

The insect cell line Spodoptera frugiperda (Sf9) was cultured in Insect Sf-900 SFM serum-free media (Invitrogen, Waltham, Massachusetts, USA) at $28^{\circ} \mathrm{C}$. Sf9 cells were co-transfected with Baculogold ${ }^{\mathrm{TM}}$ DNA (Pharmingen, San Diego, California, USA) and the recombinant vector pAcGP67A-E1 or pAcGP67A-E2, as demonstrated by the manufacturer. Several rounds of culture were performed to amplify the recombinant virus and a high viral titer stock solution was collected. To express the protein on a bigger scale, Trichoplusia niBTI-TN-5B1-4 (High Five ${ }^{\mathrm{TM}}$ ) insect cells (Invitrogen, Waltham, Massachusetts, USA) were grown in Insect $\mathrm{Hi}$-Express serum-free media previous infection with the high titer virus. High Five ${ }^{\mathrm{TM}}$ insect cells were used to express MAYV rEP1 and rEP2 after infection with the recombinant baculovirus.

\section{Purification of recombinant envelope protein (rE1) and rE2}

Typically, $1 \mathrm{~L}$ of recombinant baculovirus-infected insect cell cultures was harvested approximately $72 \mathrm{~h}$ post-infection and the cells were centrifugated at $5000 \times \mathrm{g}$ for $15 \mathrm{~min}$ to recover only the supernatant. The supernatant was dialyzed against binding buffer $\left(50 \mathrm{mM}\right.$ Tris- $\mathrm{HCl}, \mathrm{pH} 8.0,0.5 \mathrm{M} \mathrm{NaCl}$ ), loaded onto a $\mathrm{Ni}^{2+}$-nitrilotriacetic acid (Ni-NTA) agarose column 
(Qiagen, Hilden, Germany) equilibrated with the binding buffer, and washed with washing buffer $(50 \mathrm{mM} \mathrm{Tris-HCl}, \mathrm{pH}$ 8.0, 0.5 M NaCl, $50 \mathrm{mM}$ imidazole). $\mathrm{rE} 1$ and $\mathrm{rE2}$ were eluted with elution buffer (50 mM Tris- $\mathrm{HCl}, \mathrm{pH} 8.0,0.5 \mathrm{M} \mathrm{NaCl}$, $500 \mathrm{mM}$ imidazole) and the presence of purified protein was confirmed by sodium dodecyl sulfate-polyacrylamide gel electrophoresis (SDS-PAGE). The eluted glycoproteins were dialyzed with Slide-A-Lyzer (3.5 K MWCO; Thermo Scientific, Waltham, Massachusetts, USA) in phosphate buffered saline (PBS, $\mathrm{pH} 7.4$ ) overnight at $4{ }^{\circ} \mathrm{C}$, and stored in $\mathrm{PBS}(\mathrm{pH} 7.4)$ or $5 \%(\mathrm{v} / \mathrm{v})$ glycerol at $-70^{\circ} \mathrm{C}$.

\section{Sodium dodecyl sulfate-polyacrylamide gel electrophoresis (SDS-PAGE) and western blot analyses}

The supernatants were separated on NuPAGE Novex 4-12\% Bis-Tris SDS-PAGE gels in MES-SDS running buffer (Invitrogen, Waltham, Massachusetts, USA) and visualized by staining with Coomassie brilliant blue. Gel images were obtained using a gel documentation system (AE-9000 E-graph, ATTO Corp, Tokyo, Japan).

For western blot analysis, proteins were separated on SDS-PAGE gels and transferred to polyvinylidenedifluoride (PVDF) membranes using a Trans-Bolt system (Bio-Rad, Hercules, California, USA). The membranes were blocked with $5 \%$ skim milk in $1 \times$ PBS containing $0.05 \%$ Tween-20 (PBST) for one hour at $25^{\circ} \mathrm{C}$, probed with mouse anti-His-tag antibodies (Bethyl Laboratories, Inc., Montgomery, Texas, USA) at a 1:3000 dilution for one hour at $25^{\circ} \mathrm{C}$, washed six times with PBST, and incubated with HRP-conjugated goat-anti mouse IgG antibodies at a 1:10,000 dilution for $1 \mathrm{~h}$ at $25^{\circ} \mathrm{C}$. To visualize antibodies bound to the proteins, ECL luminal kits were used as enzyme substrates and the blots were visualized on a chemiluminescence imaging system (WSE-6200H LuminoGraph II, ATTO Corp, Tokyo, Japan).

\section{Mayaro antigen-specific ELISA}

rE1 and rE2 reactivity against human anti-MAYV IgG and IgM was tested (11). A 96-well microtiter plate (Costar 3690, Corning Inc., New York, USA) was coated with $100 \mathrm{ng} /$ well of purified rE1 or rE2 diluted to $1 \mu \mathrm{g} / \mathrm{mL}$ in $0.1 \mathrm{M}$ carbonatebicarbonate buffer ( $\mathrm{pH}$ 9.0) overnight at $4^{\circ} \mathrm{C}$. Unbound antigens were discarded and the wells were blocked with $2 \%$ BSA in PBS for $60 \mathrm{~min}$ at $25^{\circ} \mathrm{C}$. After washing, the wells were incubated at $37^{\circ} \mathrm{C}$ for $2 \mathrm{~h}$ with MAYV positive controls (human anti-MAYV IgG or IgM) from an anti-MAYV ELISA kit (Euroimmun, Luebeck, Germany). CHIKV positive controls (human anti-CHIKV IgG or IgM) from an anti-CHIKV ELISA kit (Euroimmun, Luebeck, Germany) were used to test cross-reactivity. The plates were washed six times with PBST and incubated at $37^{\circ} \mathrm{C}$ for $1 \mathrm{~h}$ with HRP-conjugated anti-human IgG or IgM diluted at 1:10,000. Bound antibodies were detected by adding 3, 3', 5, 5' -tetramethylbenzidine (TMB, Sigma, St. Louis, Missouri, USA) and optical density was measured at $450 \mathrm{~nm}$ using an ELISA microplate reader (Thermo Scientific, Waltham, Massachusetts, USA). Bars represent the average absorbance of three measurements and the error bars indicate standard deviations (SD) of triplicate measurements.

\section{Immunochromatographic Strip Test (IST) method}

The IST system in this study used a working solution (PBST) and test strips. Nitrocellulose (NC) membranes, laminated cards, absorbent pads, and sample pads were obtained from Millipore. Red latex beads were supplied by Expedeon. Goat anti-mouse IgG antibodies and other chemicals were purchased from Sigma-Aldrich. The strip sensor was composed of a sample pad, NC membrane, absorption pad, and a backing card. The latex beads were covalently conjugated to rE2 lysine residues, soaked onto the conjugate pad, and dried at $37^{\circ} \mathrm{C}$ for $2 \mathrm{~h}$. Anti-human $\operatorname{lgG}(0.1 \mathrm{mg} / \mathrm{mL})$ or $\operatorname{lgM}(0.1 \mathrm{mg} / \mathrm{mL})$ antibodies were immobilized on the test line and anti-His-tag antibodies $(0.1 \mathrm{mg} / \mathrm{mL})$ were deposited on the control line on a NC membrane using a dispenser (DCI100; Zeta Corporation, Gunpo-si, Gyeonggi-do, Korea). It was assembled on a backing card with a $2 \mathrm{~mm}$ overlap between each component of the strip sensor. To verify IST performance, we used MAYV positive controls (human anti-MAYV IgG and IgM) from an anti-MAYV ELISA kit (Euroimmun, Luebeck, Germany) and CHIKV positive controls (human anti-CHIKV IgG or IgM) from an anti-CHIKV ELISA kit (Euroimmun, Luebeck, Germany). 
Samples (50 $\mu \mathrm{L}$ of each) were pipetted onto the sample pad and migrated along the strip membrane by capillary action. Buffer-only (PBST with human serum albumin) was also tested as a negative control (Neg. C.trl.). For data analysis, the color signal intensity of the test lines was quantified by calculating the average pixel intensity inside the test line using a custom script in the Fujifilm multi-gauge program (Fuji photo film Co, Ltd., Tokyo, Japan). Data sets were analyzed statistically using Origin software.

\section{RESULTS}

\section{Construction of recombinant baculovirus to express MAYV rE1 and rE2 in insect cells}

To express secreted versions of MAYV rE1 and rE2, we generated two recombinant expression constructs incorporating an N-terminal gp67 secretion signal peptide for recombinant protein secretion and a C-terminal His $6 \mathrm{X}$ tag for purification. The MAYV E1 and E2 genes, 442 bp at positions 1 442 of E1 and 421 bp at positions 1 421 off E2, were inserted into the pAcGP67A vector at Smal and Xbal restriction sites, respectively (Fig. 1a and b). Sf9 insect cells were transfected with selected cloned vectors (pAcGP67A-E1 or pAcGP67A-E2 plasmid) and wild type viral DNA. Each gene was inserted into the viral genome via homologous recombination to produce recombinant baculovirus $(12,13)$.

a

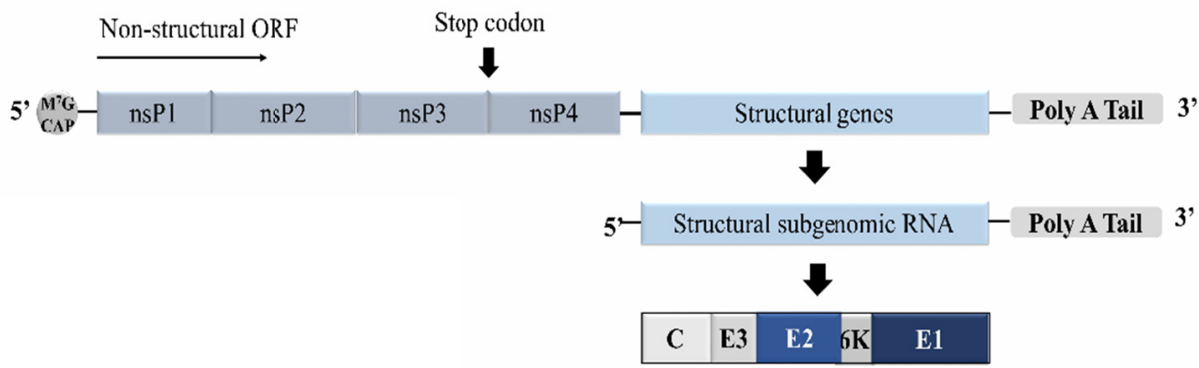

b

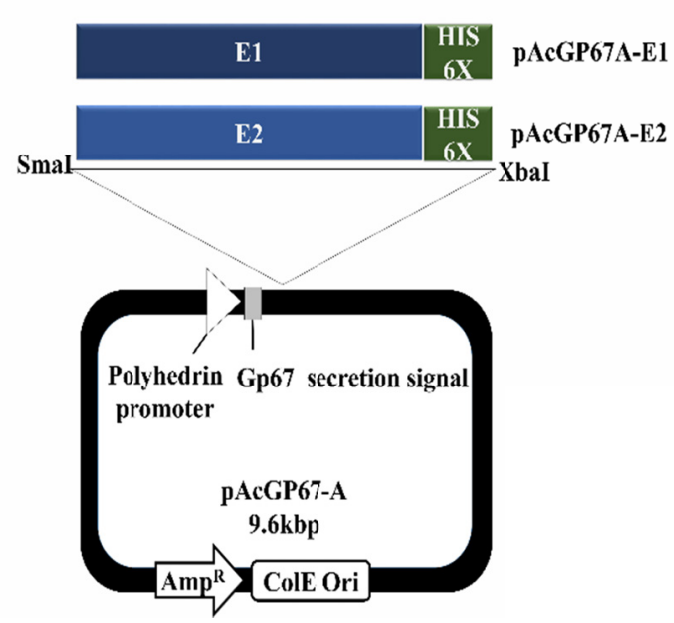

c

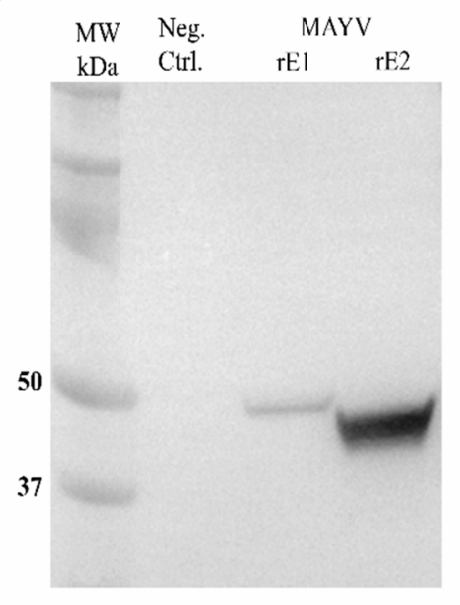

Fig. 1. MAYV rE1 and rE2 expression using a recombinant baculovirus/insect system. (a) Schematic representation of MAYV genome organization. (b) Schematic of the pAcGP67A-E1 and pAcGP67A-E2 constructs generated. Cterminal His-tags were used to purify the MAYV E1 and E2 proteins. (c) Western blot analysis of media from pAcGP67A-E1- and pAcGP67A-E2-transfected Sf9 cells using anti-His-tag antibodies conijugated to horseradish peroxidase. Uninfected Sf9 cell cultivation media was used as a negative control (Neg. Ctrl.). 
Sf9 cells were directly infected with cell culture medium containing the recombinant baculovirus to produce a high titer baculovirus stock. rE1 and rE2 expression by the infected Sf9 cells was studied on a small scalle $\left(1 \times 10^{6}\right.$ cells in a $25 \mathrm{~cm}^{2}$ flask). Western blot analyses revealed protein bands at $50 \mathrm{kDa}$ (Fig. 1C) and 48 kDa, corresponding well with the calculated molecular masses of rE1 and rE2.

\section{Overexpression and purification of MAYV rE1 and rE2 in High-Five ${ }^{\mathrm{TM}}$ insect cells by recombinant baculoviruses}

Soluble rE1 and rE2 were overexpressed by infecting a new High-Five insect cell batches with high titer recombinant baculovirus (14) and then secreted into the extracellular medium. The highest rE1 and rE2 yields were obtained $72 \mathrm{~h}$ post infection.

rE1 and rE2 secreted into the medium were purified using a Ni-NTA affinity column, which can especially bind with the Cterminal His-tag in rE1 and rE2. The proteins were eluted with binding buffer containing $500 \mathrm{mM}$ imidazole, analyzed by SDS-PAGE (Fig. 2a), and their concentrations were established using Bradford assays. The total yields of MAYV rE1 and $\mathrm{rE} 2$ were $0.2 \mathrm{mg} / \mathrm{L}$ and $2 \mathrm{mg} / \mathrm{L}$ at a concentration of $1 \times 10^{7}$ High-Five $^{\mathrm{TM}}$ cells $/ \mathrm{mL}$. The $\mathrm{Ni}^{2+}$ affinity purification of secreted rE1 and rE2 resulted in a protein recovery of $\sim 60 \%$ and purity of $95 \%$. The purified proteins were recognized by immunoblot analysis using the anti-His6X antibodies (Fig. 2b).

\section{a}

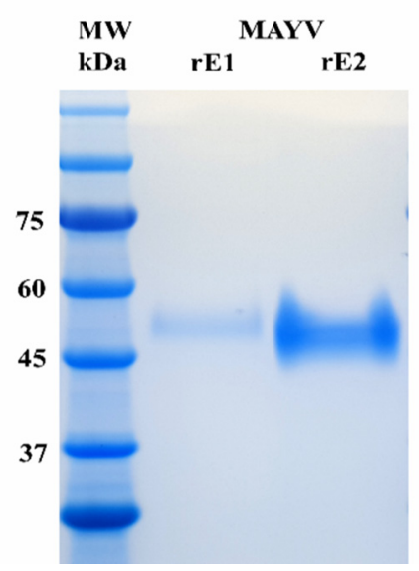

b

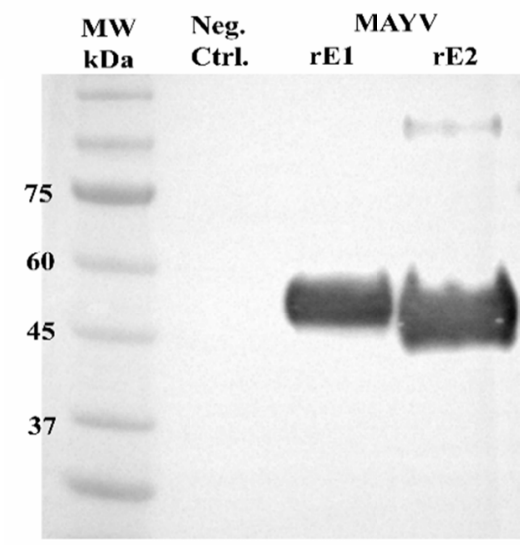

Fig. 2. Analysis of MAYV rE1 and rE2 overexpression and purification by High Five ${ }^{\text {TM }}$ cells. (a) SDS-PAGE of purified MAYV rE1 and rE2. The gel was stained with Coomassie brilliant blue R-250. (b) Western lblot analysis of purified MAYV rE1 and rE2. After being transferred to a PVDF membrane, the proteins were detected by anti-His-tag antibodies conjugated to horseradish peroxidase. Uninfected High-Five ${ }^{T M}$ insect cell cultivation media was used as the Neg. Ctrl.

\section{Antigenic rE1 and rE2 characterization}

To assess the antigenicity of purified $\mathrm{rE} 1$ and $\mathrm{rE2}$, we measured their antigenic reactivity using a modified ELISA (Euroimmun, Luebeck, Germany) with positive (human anti-MAYV IgG or $\lg M$ ) and negative controls from the anti-MAYV ELISA kit. Human anti-CHIKV IgG or IgM ELISAs (Euroimmun, Luebeck, Germany) were used to evaluate cross-reactivity with the rE1 and rE2 MAYV ELISAs. 
As shown in Fig. 3, rE1 and rE2 exhibited significantly higher reactivity with human anti-MAYV IgG and IgM than the Neg. Ctrl. (BSA). IgG and IgM ELISAs indicated that rE1 and rE2 reacted with anti-MAYV IgG and IgM; rE1 showed a specific response but a very low signal value, whilst rE2 showed higher reactivity than $\mathrm{rE1}$. Additionally, rE1 displayed low reactivity with anti-CHIKV IgG (Fig. 3a), but rE2 showed no cross-reactivity in the anti-CHIKV' IgG or IgM ELISAs. These results indicate that both proteins could react with anti-MAYV IgG and IgM in infected sera; however, rE2 was more reactive than $\mathrm{rE} 1$ with anti-MAYV IgG and IgM and showed no cross-reactivity for anti-CHIKV IgG and IgM. Therefore, we concluded that rE2 would be more suitable for MAYV diagnosis than rE1.

\section{Immunochromatographic Strip Test (IST) using human MAYV IgG and IgM}

To estimate the ability of the IST to detect human anti-MAYV IgG and IgM, we developed a MAYV rE2 antigen strip for MAYV detection. As described in Fig. 4a, the IST consisted of a backing plate, absorption pad, NC membrane, conjugate pad, and sample pad. Red latex beads were labeled with purified rE2 and loaded onto the conjugate pad. Anti-human IgG or IgM and anti-His-tag antibodies were immobilized on the test and control lines, respectively.

a
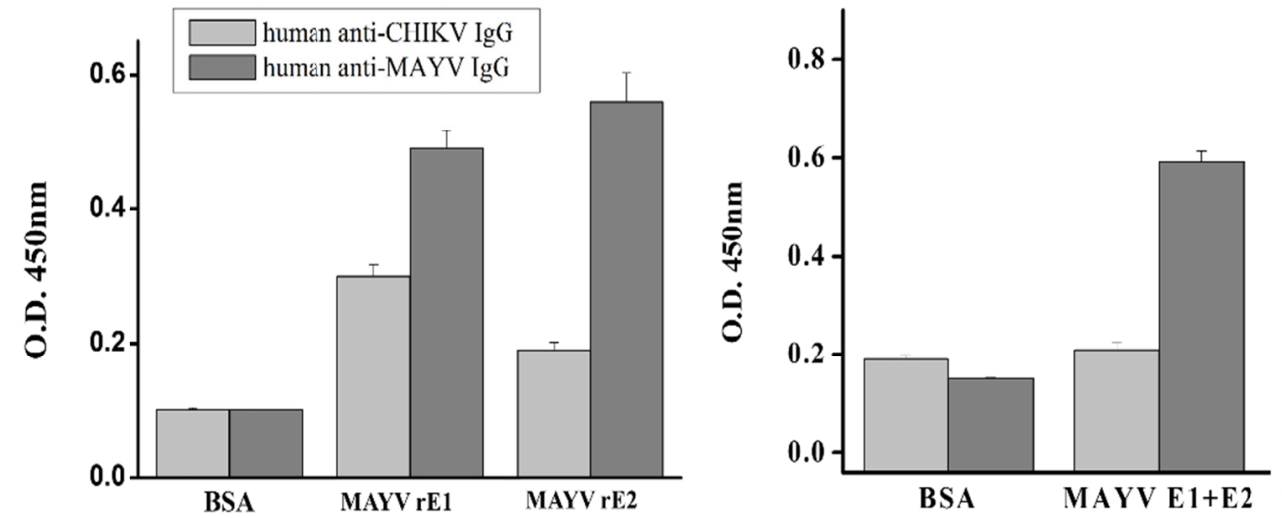

b
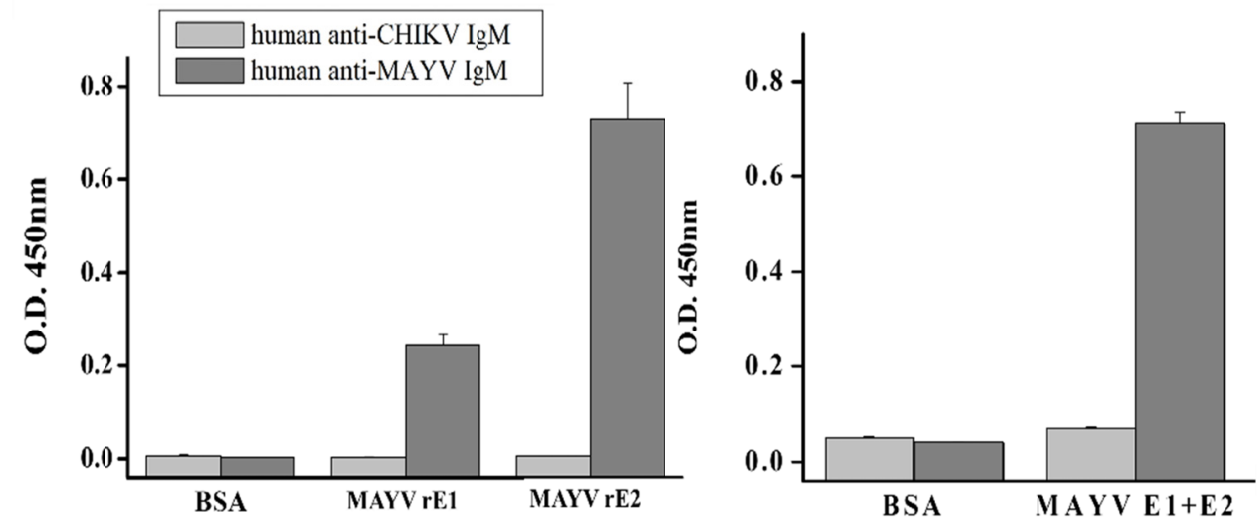

Fig. 3. rE1 and rE2 reactivity against human anti-MAYV IgG (a) and IgM (b). rE1 and rE2 cross-reactivity were determined by ELISA using anti-MAYV IgG and IgM or anti-CHIKV IgG and IgM. Each microplate well was coated with $100 \mathrm{ng}$ of purified recombinant proteins. Bars indicate the mean absorbance at $450 \mathrm{~nm}$. Error bars indicate standard deviations (SD) of triplicate measurements. 
First, samples including human anti-MAYV IgG or IgM were dropped onto the sample pad. After the sample had moved to the conjugate pad, human anti-MAYV IgG or IgM in the sample captured red latex beads labeled with rE2 to produce target complexes. When the target complexes flowed across the test line, they were captured by anti-human IgG or IgM. Complex accumulation produced a visible color on the test line. Free red latex beads labeled with rE2 flowed across the control line and were captured by anti-His-tag antibodies (Fig. 4b). To verify IST performance, we used MAYV (human anti-MAYV IgG and IgM) and CHIKV (human anti-CHIKV IgG or IgM) positive controls from the anti-MAYV and antiCHIKV ELISA kits (Euroimmun, Luebeck, Germany), respectively. Human anti-MAYV IgG and IgM caused a band to appear on the test line of the IST within 15 min (Fig. 4c). No nonspecific bands appeared on the test line and human anti-CHIKV IgG and IgM were tested simultaneously. Positive bands were only observed in the MAYV positive sample.

a

\section{Test line Control line}

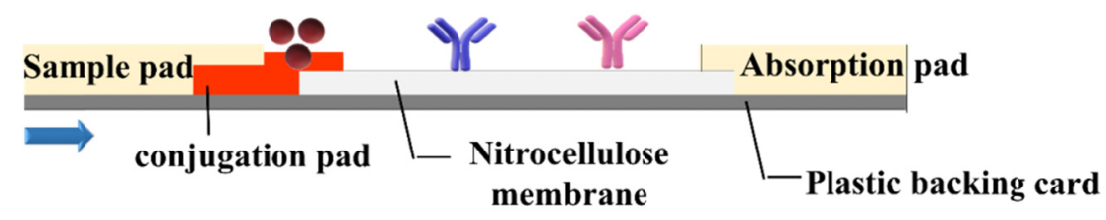

b
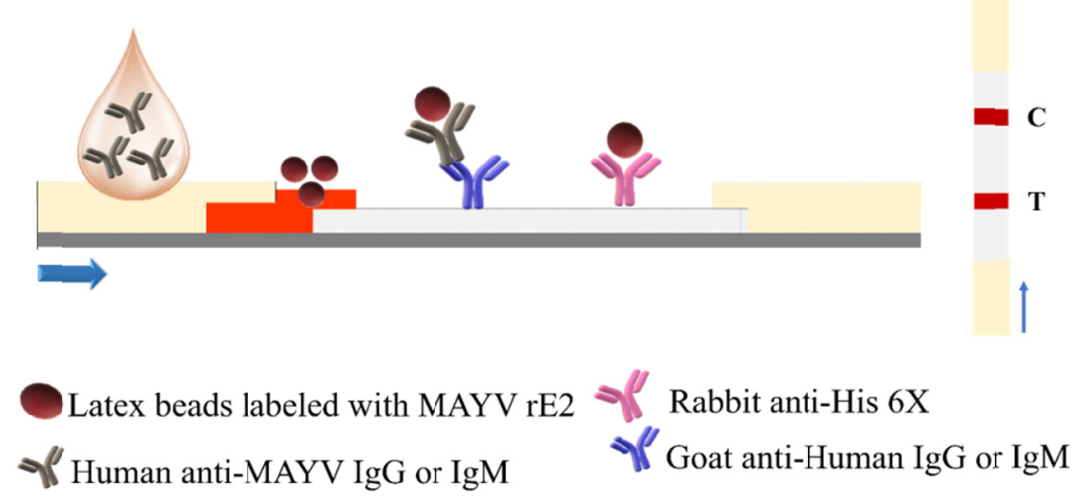

c
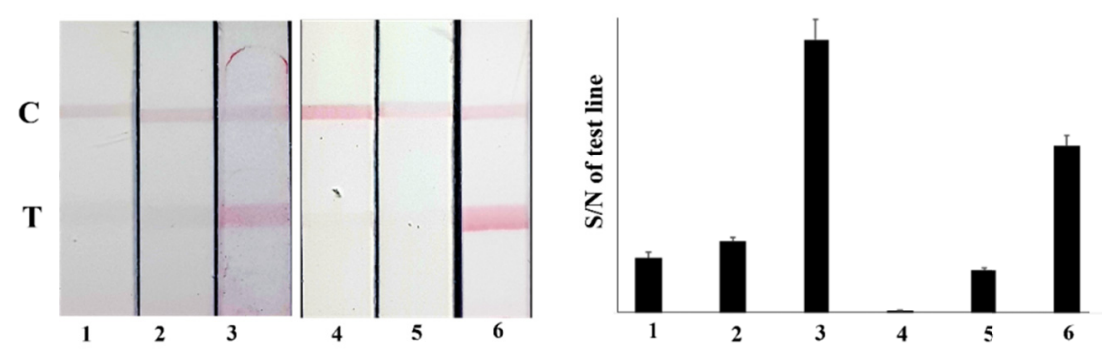
1. Neg. Ctrl.
4. Neg. Ctrl.
2. Human anti-CHIKV IgG
5. Human anti-CHIKV IgM
3. Human anti-MAYV IgG
6. Human anti-MAYV IgM

Fig. 4. Schematic illustration of the MAYV IST assay $(a, b)$ and an image of MAYV IST using rE2 to detect human anti-MAYV IgG and IgM (c). BSA was used as a Neg. Ctrl. Quantification of band intensity at the strip test line. Normalized pixel intensity was quantified using Image software. Intensity values were calculated relative to the control line intensity. The graph presents the ratio of signal to noise $(\mathrm{S} / \mathrm{N})$ on the test line. 


\section{DISCUSSION}

Mayaro virus is an emerging epidemic prevalent in tropical regions of South America; its range has recently spread into Central America and Caribbean island nations (15). The virus causes acute febrile illness and can cause long term joint pain in some patients after infection. Currently, there are no permitted vaccines or treatments for MAYV infection and transmission (4). MAYV is diagnosed by virus genome-wide detection analysis using viral isolation or a viral standard: however, this method is limited to viremic phase plasma which usually lasts no longer than 5 days (16).

Recently, the envelope proteins of alphaviruses have been studied to develop a method of MAYV diagnosis (17). MAYV encodes envelope glycoproteins; the E1 protein is primarily required in cell fusion, E2 binds to receptors of cell for entry. $E 1$ and E2, surface protein, are significant targets for neutralizing antibodies in the host immune response against most alphaviruses (2). Until now, serological assays for commercial MAYV diagnosis have not been available.

In this study, we expressed and purified MAYV rE1 and rE2 in a baculovirus expression system as efficient antigens for serological diagnosis. The $E 1$ and $E 2$ genes of MAYV were cloned into a pAcGP67A vector that used the gp67 secretory signal sequence of the baculovirus protein to secrete the recombinant proteins. The six coding codons of histidine residues downstream of the $E 1$ and E2 genes were translated into polyhistidine peptides at the C-terminals of E1 and E2 for protein purification. Insect cell expression systems are often used for mass production of transformatively modified biologically active proteins. 48 hours after infection, viral infected cells produced a small amount of proteins recognized by anti-His 6X antibodies; the major one had a molecular weight of $50 \mathrm{kDa}$. When High Five ${ }^{\mathrm{TM}}$ cells were infected, these proteins were nearly entirely secreted into the extracellular medium, with the highest levels obtained 3 days post-infection and decreasing thereafter due to proteases from lysed cells (18). Thus, we obtained rE1 and rE2 secreted into the extracellular medium for $72 \mathrm{~h}$ using High Five ${ }^{\mathrm{TM}}$ insect cells. Recombinant His-tagged rE1 and rE2 proteins were successfully eluted from the column. rE1 and rE2 purity were found to exceed $95 \%$ in the reducing condition. The yield was $0.2 \mathrm{mg}$ and $2 \mathrm{mg}$ of rE1 and rE2 per liter of media, respectively, with rE1 yield much lower than rE2 yield. Smith JL, et. al reported that MAYV rE1 and rE2 are expressed as inclusion bodies in bacterial expression system (2). However, to our knowledge, no studies have reported the expression and purification of MAYV rE1 and rE2 in insect cells.

Because infected patient samples were difficult to obtain, purified rE1 and rE2 were tested with MAYV positive controls from human anti-MAYV IgG and IgM ELISA kits (Euroimmun) to confirm their reactivities. The rE1 and rE2 ELISAs were able to detect human anti-MAYV IgG and IgM; in particular, rE2 was more reactive than rE1. Cross-reactivity between related alphaviruses is a major concern in serological diagnostic analysis (19). To solve this common problem, we measured cross-reactivity against human anti-CHIKV antibodies. As shown in Fig. 3, rE2 showed no cross-reactivity with anti-CHIKV IgG/IgM, whilst rE1 exhibited low reactivity with human anti-CHIKV IgG. Therefore, rE2 MAYV ELISA is a suitable tool for diagnosing MAYV infection which can be performed on acute serum samples and can be used for serological investigations

The IST analysis is a recognized POCT (point-of-care testing) technique. The method enables inexpensive and rapid analysis by users (20). As shown in Fig. 4, the principle of the strip sensor was based on the immune system (antigenantibody reaction) between anti-human IgM and $\lg G$ (in the test line) and red latex beads labeled with rE2 complex human anti-MAYV IgG and IgM (in samples). When the sample was loaded on the strip, human anti-MAYV IgG or IgM in the samples captured red latex beads labeled with rE2 to form a target complex. During the sample moved to the end of the strip, the target complex is combined with capture antibodies on the test line to form a red signal on the test line. Free red latex beads labeled with rE2 were captured by anti-His-tag antibodies on the control line. We concluded that the strip sensor had no interaction with CHIKV. All results from the IST were checked by ELISA, and the results of the two analysis methods were relatively well matched.

In conclusion, we expressed purified MAYV rE1 and rE2 in insect cells and showed that MAYV ELISA and an IST assay 
based on rE1 or rE2 could be useful tools for detecting human anti-MAYV IgG without cross-reactivity with CHIKV, which may be useful for serological screening during possible MAYV outbreaks. Thus, we believe that MAYV rE1 and rE2 have great potential as diagnostic reagents for detecting MAYV infection.

\section{ACKNOWLEDGMENTS}

This study was supported by BioNano Health Guard Research Center, funded by the Ministry of Science and ICT (MSIT) of Korea as a Global Frontier Project (Grant number H-GUARD_2013 M3A6B2078950).

\section{REFERENCES}

1) Muñoz M, Navarro JC. [Mayaro: a re-emerging Arbovirus in Venezuela and Latin America]. Biomedica 2012;32:286302.

2) Smith JL, Pugh CL, Cisney ED, Keasey SL, Guevara C, Ampuero JS, et al. Human Antibody Responses to Emerging Mayaro Virus and Cocirculating Alphavirus Infections Examined by Using Structural Proteins from Nine New and Old World Lineages. mSphere 2018;3.

3) Suhrbier A, Jaffar-Bandjee MC, Gasque P. Arthritogenic alphaviruses--an overview. Nat Rev Rheumatol 2012;8:420-9.

4) Choi H, Kudchodkar SB, Reuschel EL, Asija K, Borole P, Ho M, et al. Protective immunity by an engineered DNA vaccine for Mayaro virus. PLoS Negl Trop Dis 2019;13:e0007042.

5) Powers AM, Aguilar PV, Chandler LJ, Brault AC, Meakins TA, Watts D, et al. Genetic relationships among Mayaro and Una viruses suggest distinct patterns of transmission. Am J Trop Med Hyg 2006;75:461-9.

6) Rodríguez-Morales AJ, Paniz-Mondolfi AE, Villamil-Gómez WE, Navarro JC. Mayaro, Oropouche and Venezuelan Equine Encephalitis viruses: Following in the footsteps of Zika? Travel Med Infect Dis 2017;15:72-3.

7) Auguste AJ, Liria J, Forrester NL, Giambalvo D, Moncada M, Long KC, et al. Evolutionary and Ecological Characterization of Mayaro Virus Strains Isolated during an Outbreak, Venezuela, 2010. Emerg Infect Dis 2015;21: 1742-50.

8) Tesh RB, Watts DM, Russell KL, Damodaran C, Calampa C, Cabezas C, et al. Mayaro virus disease: an emerging mosquito-borne zoonosis in tropical South America. Clin Infect Dis 1999;28:67-73.

9) Esposito DLA, Fonseca BALD. Will Mayaro virus be responsible for the next outbreak of an arthropod-borne virus in Brazil? Braz J Infect Dis 2017;21:540-4.

10) Cheng RH, Kuhn RJ, Olson NH, Rossmann MG, Choi HK, Smith TJ, et al. Nucleocapsid and glycoprotein organization in an enveloped virus. Cell 1995;80:621-30.

11) Tello D, Rodríguez-Rodríguez M, Yélamos B, Gómez-Gutiérrez J, Peterson DL, Gavilanes F. High-yield production of a chimeric glycoprotein based on permuted E1 and E2 HCV envelope ectodomains. J Virol Methods 2015;213:38-44.

12) Masoomi Dezfooli S, Tan WS, Tey BT, Ooi CW, Hussain SA. Expression and purification of the matrix protein of Nipah 
virus in baculovirus insect cell system. Biotechnol Prog 2016:32:171-7.

13) Tello D, Rodríguez-Rodríguez M, Yélamos B, Gómez-Gutiérrez J, Ortega S, Pacheco B, et al. Expression and structural properties of a chimeric protein based on the ectodomains of E1 and E2 hepatitis $C$ virus envelope glycoproteins Protein Expr Purif 2010;71:123-31.

14) Lombana L, Ortega-Atienza S, Gómez-Gutiérrez J, Yélamos B, Peterson DL, Gavilanes F. The deletion of residues 268-292 of E1 impairs the ability of HCV envelope proteins to induce pore formation. Virus Res 2016:217:63-70.

15) Mackay IM, Arden KE. Mayaro virus: a forest virus primed for a trip to the city? Microbes Infect 2016;18:724-34.

16) Pinheiro FP, Freitas RB, Travassos da Rosa JF, Gabbay YB, Mello WA, LeDuc JW. An outbreak of Mayaro virus disease in Belterra, Brazil. I. Clinical and virological findings. Am J Trop Med Hyg 1981;30:674-81.

17) Fumagalli MJ, de Souza WM, Romeiro MF, de Souza Costa MC, Slhessarenko RD, Figueiredo LTM. Development of an Enzyme-Linked Immunosorbent Assay To Detect Antibodies Targeting Recombinant Envelope Protein 2 of Mayaro Virus. J Clin Microbiol 2019;57.

18) Rodríguez-Rodríguez M, Tello D, Yélamos B, Gómez-Gutiérrez J, Pacheco B, Ortega S, et al. Structural properties of the ectodomain of hepatitis C virus E2 envelope protein. Virus Res 2009;139:91-9.

19) Powers AM, Brault AC, Shirako Y, Strauss EG, Kang W, Strauss JH, et al. Evolutionary relationships and systematics of the alphaviruses. J Virol 2001;75:10118-31.

20) Song C, Li J, Liu J, Liu Q. Simple sensitive rapid detection of Escherichia coli O157:H7 in food samples by label-free immunofluorescence strip sensor. Talanta 2016;156-157:42-7. 